\title{
El paciente del siglo XXI
}

\section{The $X X I$ century patient}

\author{
A. J. Jovell
}

\section{RESUMEN}

Estamos viviendo un cambio social en la sanidad sin precedentes en la historia de la humanidad. La sociología moderna intenta explicar este cambio mediante los conceptos de modernización reflexiva, vida o sociedad líquida o fin de la historia. Este cambio social se traduce en un nuevo modelo de pacientes más informado y con unas mayores expectativas con respecto a la sanidad y a la salud. Mayor información no supone mejor conocimiento y más responsabilidad sobre la salud. Para ello se requiere aumentar la consciencia social e individual de las personas como agentes de salud y como usuarios responsables. La Universidad de los pacientes aparece como proyecto orientado a aumentar la alfabetización sanitaria y cívica de la población y como necesidad de adaptar los sistemas sanitarios a las nuevas necesidades generadas por un nuevo modelo de usuario.

Palabras clave. Cambio social, empoderamiento paciente, expectativas usuario, confianza, paciente del futuro

An. Sist. Sanit. Navar. 2006; 29 (Supl. 3): 85-90.

\begin{abstract}
We are experiencing a social change in health that is unprecedented in the history of humanity. Modern sociology attempts to explain this change using concepts of reflexive modernisation, liquid life or society, or the end of history. This social change results in a new model of patient who is better informed and has greater expectations with respect to healthcare and health. More information does not mean better understanding and more responsibility with respect to health. For this to occur, it is necessary to increase the social and individual conscience of people as health agents and as responsible users. The University of the Patients emerges as a project directed towards increasing the health and civic literacy of the population and from the need to adapt the health systems to the new needs generated by a new model of user.
\end{abstract}

Key words. Social change. Patient empowerment. User expectations. Trust. Patient of the future.
Director General Fundació Biblioteca Josep Laporte. Facultad de Medicina. Universitat Autònoma de Barcelona

\section{Correspondencia:}

E-mail: albert.jovell@uab.es www.fbjoseplaporte.org 


\section{LA SOCIEDAD CAMBIA MÁS RÁPIDO QUE LA SANIDAD}

La sociedad del siglo XXI cambia de forma rápida en dirección incierta. El sociólogo Zygmunt Bauman ha acuñado el concepto de sociedad o vida líquida para definir un modelo emergente de sociedad en el que las cosas se convierten en objetos de consumo o en objetos de reciclaje, y en la que nunca se sabe con certeza hacia dónde se evoluciona ${ }^{1}$. En este mismo contexto, el sociólogo Ulrick Beck ha hablado de la sociedad del riesgo como aquella en la que la búsqueda de certezas genera más incertidumbre ${ }^{2}$. Finalmente, Francis Fukuyama se ha referido al fin de la historia para definir un modelo de sociedad en la que los acontecimientos pasan con gran rapidez, de forma inmediata y son objetos de múltiples análisis en tiempo presente ${ }^{3}$. Este proceso de finitud de la historia, como disciplina encargada de la descripción y el análisis retrospectivo de los acontecimientos sociales, es consecuencia de un conjunto de fenómenos que, en lo que respecta a los sistemas sanitarios, se pueden clasificar como "transiciones sanitarias" (Tabla 1) y que permiten la visualización de los cambios conforme éstos se van produciendo, en "tiempo real".

Tabla 1. Transiciones sanitarias determinantes del cambio social.
- Demográfica
- Epidemiológica
- Económica
- Educativa
- Laboral
- Tecnológica
- Mediática
- Judicial
- Política
- Ética

Este concepto de now-history o "historia en tiempo real" dificulta la capacidad de reacción de los agentes sanitarios, a los que los determinantes del cambio suelen pillarles desprovistos de soluciones. Ello es debido, en parte, a que la velocidad del cambio es mayor que la capacidad de reacción al mismo por parte de las personas e instituciones sanitarias. Un ejemplo de esta situación lo constituye el aumento de tarjetas sanitarias que se ha producido en algunas CC.AA. y que ha supuesto un colapso de algunos servicios sanitarios debido a una demanda asistencial no prevista. A ello se une la escasa capacidad de planificación de los servicios sanitarios, como se ha demostrado con la imprevisión producida en la estimación del número de médicos necesarios. La coexistencia en el tiempo de un aumento del número de jubilaciones de profesionales con la disminución del número de nuevos licenciados en activo -resultante de las políticas de numerus clausus que se han aplicado desde 1980 en las facultades de medicina- era un fenómeno previsible que no había sido adecuadamente previsto.

Los sociólogos modernos concluyen que vivimos en la sociedad de la incertidumbre, del cambio y del exceso, y esas características también se reflejan en las actitudes de los ciudadanos con respecto a la salud y a los sistemas sanitarios. Las características más relevantes de este nuevo modelo de cambio social se describen en la tabla 2 .

Tabla 2. El nuevo modelo de usuario de los servicios sanitarios.

- Aumento del nivel de alfabetización formal

- Mayor del nivel de educación reglada

- Aumento de las necesidades de atención social y sociosanitaria

- Escasa educación cívica en relación al uso de los bienes públicos

- Cambio de la categoría de paciente a la de cliente

- Acceso a mayor información mediante Internet

Así, en España se ha configurado un nuevo modelo de usuario de la sanidad que condiciona los patrones de utilización de los servicios sanitarios y aumenta la complejidad de la visita médica. Una complejidad que, de hecho, está influenciada por una condiciones de presión asistencial, difusión rápida de nuevas tecnologías, escasa preparación de los profesionales de la medicina en habilidades de relación y comunicación con los pacientes, mayor exigencia de los usua- 
rios, baja autoestima y burn-out de los profesionales de la atención médica, y unas elevadas expectativas en la capacidad resolutiva de los servicios de salud y en el beneficio potencial de los avances científicos.

En España se ha producido en los últimos años una elevada alfabetización de la población, entendida ésta como un aumento del porcentaje de ciudadanos que saben leer, escribir y hacer operaciones numéricas simples. A ello se ha unido un incremento de la educación formal de la ciudadanía, medida por el porcentaje de personas que han finalizado los estudios de bachillerato y los que han tenido acceso a estudios universitarios. Curiosamente, este aumento de la alfabetización formal básica y superior no se ha acompañado de un incremento de la alfabetización sanitaria y de la educación cívica. Por alfabetización sanitaria se entiende la capacidad de las personas para encontrar, entender y utilizar adecuadamente, en beneficio propio o de aquellas personas que cuidan, la información relacionada con los temas de salud. Asimismo, el concepto de educación cívica hace referencia, en este caso, a la capacidad de los ciudadanos para realizar un uso justificado y adecuado de los recursos públicos. A estos déficits de alfabetización sanitaria y educación cívica se une la extensión, en el Estado del bienestar, de los servicios sanitarios al ámbito de las políticas sociales. Esta situación se manifiesta en tres situaciones específicas: los centros de salud, los servicios de urgencias y la atención sociosanitaria.

En los sistemas sanitarios modernos el centro de salud se convierte en un "centro social" ya que parte de la patología que atiende está relacionada o acompañada de situaciones relativas a la dependencia, problemas de salud mental, estrés, soledad y adicciones, entre otras condiciones de naturaleza social. Ello ha llevado a algunos sistemas sanitarios a considerar al centro de salud como un centro social o sociosanitario, lo que supondría, en términos de organización de la asistencia, dar una mayor relevancia de la que tienen en la actualidad a los profesionales de enfermería y a los trabajadores sociales.
Otra característica singular de nuestro sistema sanitario es la utilización que se hace de los servicios de urgencias. En ésta se ve el reflejo de una sociedad que no sabe esperar y que busca encontrar soluciones rápidas a los problemas de salud. El hecho de que las urgencias hospitalarias sea la puerta preferida por muchos ciudadanos para entrar en el sistema sanitario cuando tienen un problema de salud indica, en aquellos casos en los que no constituye la alternativa adecuada, un déficit de educación cívica sobre los usos de los bienes públicos y un problema de alfabetización sanitaria. A ello se une la ausencia de servicios de salud que atiendan a los pacientes en horario de tarde-noche y de fin de semana. Finalmente, el envejecimiento de la población produce un incremento de la comorbilidad, lo que se define, en la denominada transición epidemiólogica, como la transición de un estadio de enfermedad única y aguda, a uno de pluripatología y enfermedad crónica. Todo ello en un entorno sanitario donde predomina el hospital de alta tecnología y la fragmentación por especialidades, obviando una aproximación más cercana a las necesidades de salud y sociales de los pacientes.

El escenario expuesto se hace más complejo cuando la atención sanitaria califica a sus usuarios como clientes y no como pacientes. La naturaleza del concepto de cliente o consumidor implica una capacidad de elección de lo servicios por parte de éste, una participación del usuario en la evaluación de los servicios obtenidos, dar prioridad a la visión subjetiva de la necesidad médica y la adopción de un modelo de relación entre profesionales y usuarios más simétrico, entre otras tendencias emergentes. La diferencia esencial es que un consumidor de un servicio convencional, por ejemplo un supermercado, puede decidir si acude o no a éste, cuándo va y qué va a comprar, mientras que un usuario de los servicios sanitarios se supone que acude a los mismos por necesidad, no por voluntad, y su capacidad de elección está restringida. En este sentido, en los servicios de atención sanitaria se manifiesta la tensión existente entre una organización diseñada para atender enfermos de forma gratuita y universal y una concepción del paciente como 
usuario, cliente o consumidor. Ello configura la necesidad de determinar un nuevo marco de derechos y obligaciones acordes con un nuevo modelo de paciente.

Finalmente, en la denominada sociedad de la información, Internet ha supuesto una verdadera revolución. Se ha pasado de una situación de incapacidad para acceder a información sobre temas de salud o del acceso a una información de escasa calidad y genérica, a acceder a una gran cantidad de información de calidad desigual y que supera la capacidad de cribado de los internautas. Así, en el mes de octubre de 2006 se podían encontrar 300 millones de referencias sobre cáncer en el buscador Google, de las que más de 3 millones eran de cáncer de mama y 1.390.000 sobre el tratamiento de este tipo de tumores con un nuevo fármaco, el Herceptin. Curiosamente, o no, Internet permite acceder a las búsquedas más variopintas, como la de encontrar 455 webs que hacían referencia al inexistente tratamiento de la angina de pecho con frijoles.

\section{La experiencia de los pacientes en España}

En los últimos años, desde la Fundación Biblioteca Josep Laporte se han dirigido y promovido diferentes estudios genéricos en pacientes y usuarios de la sanidad con los siguientes objetivos ${ }^{4}$ : valorar la percepción y experiencia de los usuarios y pacientes con el Sistema Nacional de Salud; evaluar los cambios producidos en el sistema sanitario; visualizar tendencias en la utilización de los servicios sanitarios y en el auto-cuidado; estudiar la confianza de los ciudadanos en el sistema de salud y revisar el estado de la opinión pública sobre los temas de salud y sanidad. Los estudios realizados seguían metodologías cuantitativa y cualitativa. Entre las investigaciones cuantitativas destacan: una encuesta realizada en julio 2001 para el Observatorio de Salud y Mujer en 6.530 mujeres españolas valorando sus necesidades de información de salud; otra encuesta llevada a cabo en septiembre de 2002 en una muestra de 2.700 usuarios de la sanidad y una tercera realizada en noviembre de 2005 con la Universidad de
Harvard en 3.010 ciudadanos. Entre los trabajos cualitativos, destacan: el estudio internacional "El Paciente del Futuro" que dirigido desde el Picker Institute de Oxford se realizó en 8 países europeos con la metodología de grupos focales, el estudio "El Paciente Oncológico", y estudios en pacientes con osteoporosis y con enfermedades cardiovasculares. Los principales resultados de los estudios cuantitativos aparecen resumidos en las tablas 3 y 4 .

Tabla 3. Resultados estudio OBSYM.

- 3 de cada 4 mujeres entrevistadas considera la información que obtiene en temas de salud como escasa o insuficiente.

- 3 de cada 4 mujeres que acceden a información sanitaria sobre temas de salud tiene problemas para entender el contenido de la misma.

- El cáncer es el problema de salud que más preocupa a las mujeres españolas.

- El interés por los temas de salud está relacionado con la edad, predominando entre las mujeres jóvenes y las madres de familia de hijos pequeños.

- La mujer es el agente de salud de la familia, lo que le lleva a priorizar la salud de los demás antes que la suya.

- Existe un interés creciente por acceder a información de calidad contrastada e inteligible sobre temas de salud.

Tabla 4. Resultados encuesta sanitaria del año 2002.

- El médico es la principal fuente de información de los pacientes, siendo los medios de comunicación la segunda en importancia.

- Sólo un 30\% de la población busca medios alternativos de información a los proporcionados en la consulta médica.

- $\quad$ El 50\% de los entrevistados no plantea dudas, discrepancias o preguntas específicas a los médicos.

- $\quad$ El 70\% de los entrevistados no conocen sus derechos como pacientes.

- Menos de un 5\% de los entrevistados pertenece a algún tipo de asociación que puede tener relación con temas de salud. 
- La población exige acceso a información de calidad contrastada que sea inteligible y proporcionada por profesionales.

- Se confirma la existencia de una doble tendencia en la actitud de los pacientes: pasiva y activa.

- El paciente activo suele ser joven y con mayor nivel de estudios, por lo que aparece como una tendencia emergente y de futuro.

En ellos se pone en evidencia la escasa implicación de los pacientes en la toma de decisiones sanitarias, donde adoptan mayoritariamente un rol pasivo, la necesidad de mejorar los procesos de información a los pacientes -tanto en cantidad como en calidad- y la importancia del médico y de los medios de comunicación como agentes de información. Los resultados del estudio de "Confianza en el Sistema Nacional de Salud" ${ }^{5}$ evidencian la elevada confianza de la población en las profesiones sanitarias con respecto a otras profesiones. También ponen de manifiesto que la población es consciente de que la sanidad atraviesa problemas financieros o de sostenibilidad, pero, a pesar de ello, no están dispuestos a aceptar medidas de financiación complementaria -basadas en el copago de servicios o en la subida de impuestos- a excepción de los impuestos del tabaco y del alcohol.

Los principales resultados del estudio español del proyecto internacional "El Paciente del Futuro" aparecen descritos en la tabla 5 .

Tabla 5. El paciente del futuro: Resultados en España.

- $\quad$ Exigencia de un trato más personalizado en la visita médica: mayor dedicación de tiempo y más información.

- Masificación como problema.

- Necesidad de una mejor relación médico-paciente.

- Internet sólo es valorado de forma positiva como instrumento de gestión de procesos burocráticos.

- Escasa atención al tema de los derechos de los pacientes.

- No existe una visión pesimista en torno al futuro del sistema sanitario ni con respecto a los problemas de sostenibilidad económica del mismo.
Los mismos evidencian cómo los españoles sitúan la sanidad y los temas de salud como prioritarios. Este dato confirma los resultados obtenidos en las diferentes encuestas realizadas a principios de la década de los 90 en España. También destaca la reivindicación de la figura de "mi médico" como agente de salud de los pacientes, a pesar de que se le pide una mayor dedicación en tiempo de visita y en la calidad de la información proporcionada. Esta visión se confirma en el estudio de "Confianza". Asimismo, aparece la dicotomía pasiva-activa en la actitud de los pacientes hacia los temas de salud que, posteriormente, se confirma en los estudios cuantitativos. La tendencia pasiva de los pacientes exige el protagonismo de los médicos en los procesos de toma de decisiones, adoptando un modelo de relación paternalista. Por el contrario, la tendencia activa, minoritaria en los estudios cualitativos y cuantitativos, evidencia la aparición emergente de un nuevo tipo de pacientes que quieren participar y adoptar un rol protagonista en los procesos de toma de decisiones que afectan a su salud. Estos pacientes optarían por un modelo de relación médico-paciente deliberativo e incluso, en algunos casos, científico. Se podría hipotetizar que los ejemplos de este nuevo modelo de paciente se visualizarían en un nivel educativo alto, pacientes jóvenes, padres de niños enfermos y en hijos de personas mayores.

Desde el año 2003 diferentes iniciativas han intentado fomentar la educación cívica y la promoción de los derechos de los pacientes en España. La primera de estas iniciativas fue la Declaración de Barcelona de las Asociaciones de Pacientes, formulada por representantes de 50 organizaciones de pacientes y usuarios del Estado español ${ }^{6}$. La difusión de la misma llevó a la constitución del Foro Español de Pacientes, que agrupa 14 grandes organizaciones, 637 asociaciones de pacientes y 267.000 miembros. El Foro está impulsando en la actualidad la creación de foros autonómicos, de los que ya existe el "Forum Català de Pacients" que agrupa 25 asociaciones y 31.000 miembros. El Foro ha organizado ya dos congresos nacionales de pacientes y se ha constituido en 
una organización de promoción de los derechos de los pacientes. Finalmente, con el objeto de promover una mayor alfabetización sanitaria de la sociedad civil y de los pacientes se ha creado la Universidad de los Pacientes. Este es un proyecto promovido por la Universitat Autònoma de Barcelona y la Fundació Biblioteca Josep Laporte que tiene como misión promover actividades de información, incluidos los "podcasts epacientes" y diferentes sitios webs, de investigación, de formación, con los programas de paciente experto y de paciente tutor, y de asesoría y acreditación de actividades.

\section{CONCLUSIONES}

Los resultados de los estudios citados en este artículo y la mayor parte de la bibliografía revisada evidencian dos grandes tendencias. En primer lugar, la aparición de un nuevo modelo de paciente-ciudadano que se quiere responsabilizar de su salud y la de su familia, y para ello adopta una conducta de consumidor de servicios en relación a la provisión sanitaria. En segundo lugar, los usuarios y los pacientes manifiestan tener una gran confianza en la profesión médica como agente principal del sistema. Esta confianza es superior a la depositada en otras profesiones sanitarias y se mantiene a pesar de las quejas en torno a la atención sanitaria recibida. En este sentido, las iniciativas de asociacionismo y de alfabetización sanitaria pueden contribuir a preparar a los pacientes y a los profesionales a confrontar los diferentes cambios que se están produciendo en los sistemas sanitarios modernos y a facilitar la transición política del Estado a la sociedad del bienestar.

\section{BIBLIOGRAFÍA}

1.BAuman Z. Vida líquida. Barcelona: Paidós, 2006.

1.BEcK U. La sociedad del riesgo. Hacia una nueva modernidad. Barcelona: Paidós, 2006.

3. FukUYAma F. The end of the history and the last man. New York: Penguin Books, 1993.

4. www.fbjoseplaporte.org y www.obsym.org

5. www.universidadpacientes.org

6. www.webpacientes.org. 